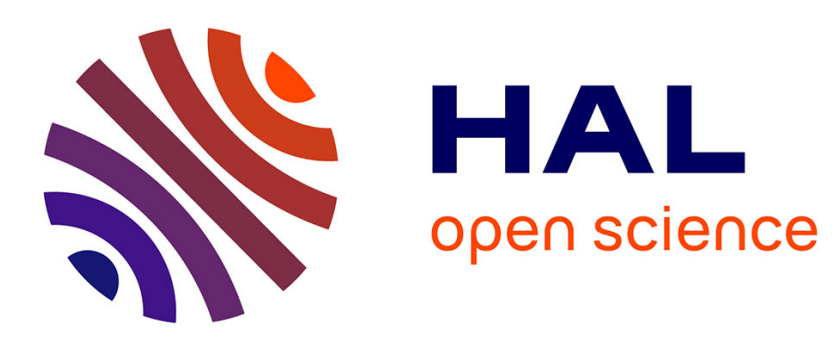

\title{
Femmes et dealers. Une recherche de terrain au sein du deal de classes moyennes bordelais.
}

Sarah Perrin

\section{To cite this version:}

Sarah Perrin. Femmes et dealers. Une recherche de terrain au sein du deal de classes moyennes bordelais.. Psychotropes, 2018, 24 (1), pp.15-37. 10.3917/psyt.241.0015 . hal-03587100

\author{
HAL Id: hal-03587100 \\ https://hal.science/hal-03587100
}

Submitted on 24 Feb 2022

HAL is a multi-disciplinary open access archive for the deposit and dissemination of scientific research documents, whether they are published or not. The documents may come from teaching and research institutions in France or abroad, or from public or private research centers.
L'archive ouverte pluridisciplinaire HAL, est destinée au dépôt et à la diffusion de documents scientifiques de niveau recherche, publiés ou non, émanant des établissements d'enseignement et de recherche français ou étrangers, des laboratoires publics ou privés. 
Une recherche de terrain au sein du deal de classes moyennes bordelais

\title{
Women and drug dealers
}

\section{A field research into Bordeaux middle class drug trafficking}

\author{
Sarah Perrin \\ Sociologue. Sciences Po Bordeaux, 11 allée Ausone, 33600 Pessac \\ 160 Cours de l'Argonne, 33000 Bordeaux sarah.perrin@scpobx.fr
}

\section{Résumé:}

Cet article propose d'étudier les situations de femmes socialement insérées dans le deal de stupéfiant bordelais. Etre une femme dans le deal implique plusieurs avantages : cela permet de tirer parti de stéréotypes positifs et d'avantages sexués à la fois dans l'achat, la vente de stupéfiants et face à la police. Mais c'est aussi s'exposer à de nombreux risques. En tant que minorité sexuée au sein d'un milieu social perçu comme dangereux, les femmes doivent prendre sans cesse prendre garde à ce que leur genre ne soit pas perçu comme un facteur de vulnérabilité physique et psychologique. Elles doivent faire face à une menace diffuse de violence et courent plus souvent le risque de se faire escroquer. Elles mettent donc en place des stratégies pour être reconnues comme des membres à part entière de cette activité illicite. Elles ressentent également un double décalage, du fait de leurs positions à cheval sur plusieurs univers sociaux: le monde des stupéfiants, et les autres sphères légitimes de leurs vies sociales.

\section{Summary :}

This article aims to study the positions of women involved in Bordeaux drug trafficking. Being a woman in drug trafficking has several benefices: it allows to women to take advantage of positive stereotypes and sexual advantages in the purchase and the sale of narcotics, and in front of the police. But it is also exposing them to many risks. As a sexual minority in a social environment perceived as dangerous, women must constantly make sure that their gender is not perceived as a factor of physical and psychological weakness. They face a widespread threat of violence and are more likely to be fooled. They therefore put in place strategies to be recognized as full members of the traffic. They also feel a double shift, because of their positions straddling several social worlds : the world of narcotics, and other legitimate spheres of their social lives. 


\section{Introduction $^{1}$}

En 2016, un article du journal Le Parisien intitulé «En Normandie, une femme de 74 ans convoyait de la drogue par amour ${ }^{2}$ » optait pour une accroche quelque peu surprenante, en commençant par une reformulation de la citation de Pascal : "L'amour a ses raisons que la raison ignore ». L'article relate l'incarcération d'une septuagénaire, arrêtée pour avoir transporté plus de 85 kilos de cannabis à travers l'Europe et qui aurait, selon son avocate, transgressé la loi « pour les beaux yeux d'un roumain élégant ». L'avocat général avait déclaré devant la chambre de l'instruction de la cour d'appel de Caen : «Quand on voit Madame pour la première fois, on comprend comment cette petite mamie retraitée a pu sillonner l'Europe sans attirer l'attention des douaniers ».

La criminalité est depuis longtemps pensée au masculin (Barbier, 2016), les femmes étant perçues comme davantage « agies » par la violence (Niget, 2012). Pour cette raison peut-être, très peu de travaux sociologiques ont étudié la place des femmes dans le trafic de stupéfiants. C'est à partir des années 80 que des recherches sociologiques issues de pays anglophones se sont penchées sur les rôles et places des femmes dans la vente et l'achat de drogues illicites. Ce changement est lié à l'augmentation des consommations de stupéfiants dans plusieurs grandes villes américaines, l'explosion du marché permettant aux femmes d'y entrer plus facilement. De fait, ces études se sont essentiellement intéressées à des femmes marginalisées, dans des contextes d'exclusion économique, sociale ou raciale (Niget, 2002 et Bourgois, 2001). Depuis, plusieurs études sur les femmes dans le deal ont été réalisées dans différents pays en ciblant des publics plus larges, notamment en Australie (Denton et O'Malley, 1999 et 2001), en Europe du Nord (Grundetjern, 2017 et Snitzman, 2007), en Nouvelle-Zélande (Hutton, 2005) et aux Etats-Unis (Ludwick, Murphy et Sales, 2015).

En France, la question des femmes dans le deal de stupéfiants n'a presque pas été abordée. Les études françaises se focalisent principalement sur le trafic de banlieue, sa division du travail, ses rapports hiérarchiques, ses phases techniques, sa violence, son vocabulaire et sa street culture (Nouguez, 2003, Kokoreff, 2011, Rachid, 2004, Copel, 2013, Ben Lakhdar, 2012). Pourtant, C. Ben Lakdhar et D. Weinberger (2011) ont annoncé un bouleversement du marché avec l'arrivée de nouveaux acteurs, issus des classes moyennes et résidant dans les zones pavillonnaires et les centres-villes. Moins violent et moins hiérarchisé, ce trafic laisse plus de place aux entrepreneurs « indépendants », étant donné son fonctionnement plus flexible. On peut donc supposer que ce type de trafic, dont les acteurs sont pour l'immense majorité insérés socialement ${ }^{3}$, laisse davantage de place aux femmes.

Dans cet article, nous nous proposons donc d'étudier les places et rôles de femmes clientes et dealeuses dans le trafic de stupéfiants bordelais. Ces femmes ne sont pas en situation de précarité, n'ont pas de grandes difficultés économiques et sont intégrées socialement ${ }^{4}$. Nous nous demanderons quels sont les avantages et les inconvénients de la position des femmes dans le deal de

\footnotetext{
${ }^{1}$ : Cet article s'inscrit dans le cadre d'une étude qualitative, en vue de la préparation d'une thèse. Merci au sociologue Emmanuel

Langlois, sans qui cet article n'aurait jamais vu le jour, au politiste Antoine Roger pour ses relectures avisées, et à tous les hommes et toutes les femmes interrogé.e.s au cours de cette étude qui ont répondu avec enthousiasme à mes questions.

2 : Trébert B, «A 74 ans, elle convoyait de la drogue par amour » - in Le Parisien - rubrique « Faits divers » (13 décembre 2016)

${ }^{3}$ : Nous entendons par « insérés socialement » le fait de suivre un cursus scolaire ou d'avoir un emploi, d'être en contact régulier avec sa famille et ses amis, d'avoir un logement non-précaire

${ }^{4}$ : Pour plus de précisions sur les caractéristiques des femmes et hommes interrogé.e.s, voir le tableau récipitulatif en Annexes.
} 
Bordeaux, quelles sont les stratégies qui en résultent et quelles compétences les femmes acquièrent à travers leurs carrières de vendeuses ou d'acheteuses de stupéfiants.

Cette recherche repose sur un corpus de 16 entretiens semi-directifs, réalisés avec 11 femmes et 5 hommes entre 2014 et 2017 résidant à Bordeaux et âgés de 21 à 29 ans- notre étude s'intéresse donc spécifiquement aux jeunes. Les individus interrogés sont tous insérés d'une manière ou d'une autre dans le monde des stupéfiants : certains sont de simples consommateurs et clients, d'autres s'intègrent dans le trafic en tant que dealer.euse.s ou revendeur.se.s. Les produits consommés et vendus sont très variés. Bien entendu, l'anonymat de tous les interrogés a été préservé. Les entretiens ont été réalisés individuellement ou en groupe, sur un mode semi-directif, pendant une à trois heures. Les individus interrogés se connaissaient parfois (Rose et Néo vivent en collocation et sont amies avec Julianne ; Alice est la grande sœur de Marina, qui connaît Nathan et Sophie...).

Dans un premier temps, nous nous attacherons à expliciter les avantages que permet la position de femme dans ce trafic de stupéfiants, et les stratégies qui leur sont liées. Puis nous aborderons les inconvénients, risques et dangers liés au fait d'être une femme dans le milieu du deal de classe moyenne bordelais, et là encore les stratégies en découlant. Enfin, en dernier lieu, nous analyserons les apprentissages retirés de l'intégration au milieu des stupéfiants, et le double sentiment de décalage exprimé par les femmes interrogées, vis-à-vis du monde de la drogue et des autres sphères sociales dans lesquelles elles sont intégrées.

\section{«Avantages » de la position de femme dans le trafic de stupéfiants}

Dans certains moments de la vie sociale, les femmes jouissent parfois de privilèges teintés de sexisme : se faire tenir la porte, se voir offrir un verre... Dans le deal également, les femmes peuvent tirer profit de leur position sexuée.

\section{«La femme » dans le deal comme objet de séduction}

Il apparaît que les femmes dans le deal sont fréquemment la cible de tentative de séduction. Ce phénomène est très peu abordé dans la littérature, mais il est récurrent dans les entretiens. Les onze interrogées affirment toutes s'être déjà fait «draguer» (Sophie, 21 ans, consommatrice et vendeuse de drogues variées) lors d'une transaction de stupéfiants avec un dealer ou un client qu'elles ne connaissaient pas. Marina (23 ans, consommatrice de cannabis et d'excitants) nous explique que «souvent, ils [les dealers qu'elle ne connaît pas personnellement] font des regards, des petits sourires ». La drogue peut devenir un argument de séduction pour le dealer : "Ça arrive même qu'ils te fassent des prix, parce qu'ils espèrent te revoir [...] A dealer égal, ça m'est déjà arrivé quand c'est un pote à moi qui y va, [...] qu'il la tope $e^{5}$ plus chère que quand moi j'y vais. » (Marina) En offrant du produit, en lui proposant un meilleur prix ou une meilleure qualité, le vendeur de stupéfiants espère s'attirer les faveurs de sa cliente. Il joue donc de sa situation de pouvoir (il possède un produit illicite et rare dont il choisit le prix de vente) en imposant une pression sexuelle à l'acheteuse.

La récurrence du phénomène peut s'expliquer par l'infériorité numérique des femmes dans le deal, et par le besoin des dealers de trouver une partenaire qui leur ressemble - leur activité étant très mal vue socialement. Comme le dit Sylvie (22 ans, consommatrice et vendeuse de cannabis), « t'as l'impression pour eux qu'une femme qui fume [...] c'est rare ». La femme dans le deal se fait

\footnotetext{
5 : «Toper » : terme issu de la toponymie psychotropique (L. Fernandes, « Acteurs et territoires psychotropiques : ethnographie des drogues dans une périphérie urbaine. », Déviance et Société, volume 26 série 4/2002 p. 427-441), qui signifie « acheter de la drogue ».
} 
donc remarquer. Au-delà de cet argument logique, les stéréotypes que peuvent avoir les hommes sur les femmes consommatrices de stupéfiants, forcément libérées, décadentes et portées sur le sexe (O. Thomas, 2013, et Fiona Hutton, 2004), jouent également.

Si ces situations sont, bien entendu, porteuses de nombreux risques, comme nous le verrons ensuite, elles peuvent aussi fournir des avantages concrets pour les femmes qui en acceptent les conditions. Ces femmes sont minoritaires parmi mes interrogées : la plupart refusent le rapport de séduction (qu'elles considèrent comme amenant à une position de "pute à came », c'est à dire une femme qui a des rapports sexuels en échange de drogues, pour reprendre les termes de Cécilia, 24 ans, consommatrice et vendeuse de cocaïne, de kétamine et de cannabis). Elles choisissent souvent de le désamorcer par l'humour («tu fais [...] une blague, et puis une tape sur l'épaule, [...] voilà, toujours sur le ton de la rigolade »- Cécilia) ou par une attitude froide et explicite. Mais quelquesunes acceptent de rentrer dans ce jeu de séduction. Elles sont conscientes des avantages qu'elles peuvent retirer de ce rapport et en adoptent donc certains codes, sans jamais aller trop loin pour ne pas donner l'impression d'être une «allumeuse ». Ainsi Alice (26 ans, consommatrice et vendeuse de cannabis, d'amphétamines et de psychotropes) explique que «c'est plus facile pour une femme assez mignonne parce que tu parles deux-trois minutes avec le mec, il va essayer de te faire des prix, limite t'en payer, donc tu vas être gagnante. »

Ce constat transparaît également dans les discours des interrogés masculins : Philippe (26 ans, consommateur et vendeur de cocaïne et de cannabis) affirme que les clientes « sont favorisées parce que c'est des meufs. [...] J'ai remarqué plusieurs fois que, une meuf, elle va chez un gars, lui il va m'allumer, il va me traiter comme de la merde et $j$ 'vais devoir aller vite, la meuf elle va se poser, elle va fumer un pét, elle va s'mettre bien ...». Ainsi, si la cliente accepte de passer un peu plus de temps avec le dealer que ne le nécessite la transaction à proprement parler, de consommer de la drogue en sa compagnie, voire de répondre à ses regards ou sous-entendus, elle pourra obtenir des avantages sur la qualité et le tarif du produit qu'elle recherche.

Bien entendu, ce jeu de séduction n'est pas sans risques. Cécilia l'explique très bien, « ça crée une ouverture ». " Allumer » le dealer tout en sachant qu'on ne lui donnera pas ce qu'il espère est un jeu dangereux.

\section{Profiter des stéréotypes de genre pour se protéger et faire face à la concurrence}

A cette vision de la femme consommatrice de stupéfiants comme objet de séduction, facilement débauchable, se juxtaposent des stéréotypes mélioratifs présents dans de nombreuses autres sphères de la vie sociale. Les femmes seraient moins violentes, plus rassurantes, plus aptes à communiquer et à être diplomates (Humbolt, 2013). Ainsi Gilles (28 ans, consommateur et revendeur de stupéfiants variés) nous explique que «quand t'achètes à une fille tu te sens un peu plus en sécurité, [...] c'est moins intimidant, [...] les femmes c'est plus doux généralement ». Certains clients masculins iront plus volontiers acheter leur produit à une dealeuse qu'à un dealer, car ils considèrent que le risque de violence physique est moindre, et par conséquent le risque d'escroquerie aussi. Cette attribution de qualités « proprement féminines » se retrouve dans d'autres secteurs professionnels (P. Chantelat, E. Bayle et C. Ferrand, 2004).

Les femmes clientes se tourneront plus encore quand elles le peuvent vers les dealeuses, très minoritaires. Josianne (26 ans, consommatrice de produits variés et revendeuse de MDMA), confirme que «les femmes qui consomment vont plus avoir tendance à aller vers une femme qui vend, ça les rassure. »Elles ont le sentiment qu'en allant acheter leur drogue à des dealeuses, non seulement le risque de violence est supprimé, mais qu'une solidarité féminine pourra se mettre en place puisque la vendeuse comme la cliente font partie de la même minorité. Parfois, cette solidarité semble en effet opérer : Cécilia et Josianne nous disent toutes les deux qu'elles ont eu l'occasion 
d'acheter de la drogue à des femmes qu'elles ne connaissaient pas et que le moment était plus détendu. Mais cette «solidarité » n'est pas automatique : Sophie s'est déjà fait escroquer par une dealeuse.

Lorsque les femmes dealeuses ont pris conscience des stéréotypes pesant sur elles, elles peuvent en jouer pour adopter des tactiques les avantageant. C'est le «feminine business model» décrit par H. Grundetjern (2016) : être attentive aux besoins de ses clients, prévenante, gentille, douce, «maternelle ». Cette stratégie est proche de celle analysée par M. D. Ludwick, S. Murphy et P. Sales (2015), qui consiste à insister sur ces traits vus comme typiquement féminins pour se démarquer de la concurrence.

Cette idée d'accentuer les stigmates de genre et de les retourner pour en tirer des avantages correspond au concept de « doing gender » (West et Zimmerman, 2009, Butler, 1990). Nous choisissons de nous rapprocher du sens donné au concept par M. D. Ludwick, S. Murphy et P. Sales (2015). Selon ces auteurs, le genre féminin peut s'utiliser dans le deal comme un outil de protection et de démarcation face à la concurrence : les femmes vont caricaturer ce qu'elles jugent être des traits féminins (s'habiller en jupe, porter des talons hauts, flirter avec le dealer ou le client, adopter une attitude dite maternelle, avoir l'air innocent...) pour se distinguer ou, au contraire, se faire discrètes. C'est ce que nous expliquent respectivement Alice et Sylvie : "la meuf qui deale on la reconnaît vite. Donc c'est avantageux ça, parce qu'on te repère grave facilement... »; "si tu cherches des clients par exemple, les gens ils sauront, ils se rappelleront, [...] au final tu sors un peu du lot des mecs qui vendent le même shit assis dehors ».

Dans les faits, les pratiques des femmes dealeuses diffèrent peu de celles des dealers, mais les représentations ne sont pas les mêmes. Notons que cette stigmatisation des femmes est, là encore, loin d'être propre au deal, et qu'elle existe, avec les gènes et les avantages qu'elle occasionne, dans des secteurs professionnels variés, licites et illicites (S. Louey et G. Schütz, 2014).

A présent, nous allons voir comment le genre féminin peut agir comme une garantie de de protection dans le deal, en analysant la discrétion des femmes face à la police.

\section{Une discrétion face à la police et un moindre risque d'incarcération}

Les femmes sont sous-représentées dans les chiffres des forces de l'ordre, concernant l'usage (91\% des individus interpelés pour usage de stupéfiants sont des hommes, Obravadovic, 2015) mais plus encore le trafic et la revente sans usage, et ce alors même que la population usagère de stupéfiants se féminise (Barbier, 2016). Les forces policières se focalisent sur un même type d'usager (les jeunes hommes d'origine maghrébine), qui est loin d'être représentatif de la diversité des profils des usagers déclarés (Obradovic, 2015 et Beck, Peretti-Watel et Legleye, 2007). Bien que plusieurs des femmes interrogées aient déjà eu des ennuis avec la police et la justice, lorsqu'elles comparent leur expérience avec celle des hommes consommateurs ou dealers qu'elles fréquentent, toutes affirment constituer bien moins une cible que leurs congénères masculins.

Plusieurs hypothèses peuvent être soulevées pour expliquer cette sous-représentation des femmes dans les usagers interpelés : les femmes auraient un usage de stupéfiants moins visibles et plus solitaires, rarement effectué dans l'espace public, ce qui diminue le risque d'interpellation (Obradovic et Beck, 2013). Mais au sein de la population étudiée, toutes les femmes interrogées disent avoir déjà été sous l'emprise de substances dans l'espace public. De plus, les femmes interrogées ne semblent pas avoir un usage plus solitaire que les hommes, nos interrogés déclarant dans l'ensemble tout autant consommer en groupe ou seul chez eux, sans qu'une distinction entre les deux sexes puisse être établie. 
Une hypothèse plus pertinente réside dans l'image que se font les forces de l'ordre des femmes, qui ne cadre pas avec la représentation qu'elles ont du dealer ou du toxicomane (Barbier, 2016). L'apparence joue énormément : Blondie (27 ans, consommatrice de cannabis et d'excitants), explique que «t'en as limite, ils ou elles cherchent à s'faire arrêter, c'est pas possible tu vois, si t'arrives avec la grosse dégaine, si c'est le sarouel pour la weed... Les dreads et tout ça, forcément...»

Même si une femme, peu importe son apparence, a moins de chance de se faire arrêter par la police qu'un homme dans l'espace public, l'habillement (sarouel, sweat-shirt à capuches, Doc Martens), la coiffure (dreadlocks, crâne rasé), ou encore le port de piercings et de tatouages sont des facteurs qui augmentent le risque d'interpellation. Il existe une tenue renvoyant à l'image du consommateur de stupéfiants, et une tenue renvoyant à l'image de la femme sage et innocente ; les femmes dans le deal en sont bien conscientes et en jouent. Ainsi Sylvie nous explique comment elle se prépare pour éviter de se faire remarquer par les forces de l'ordre : «je savais comment $m^{\prime}$ 'habiller ou me coiffer pour pas avoir de fouille, par exemple [...] $j$ 'vais m'attacher les cheveux déjà, [...] j'mets du rouge à lèvre. [...] P'tits talons, p'tite nana quoi. »

Si malgré cet avantage, une femme se fait interpeler par la police, elle peut encore et toujours bénéficier d'avantages liés aux stéréotypes de genre si elle en prend conscience et qu'elle les retourne. Nous revenons à ce concept de «doing gender»: toutes les interrogées s'accordent pour dire que « faire la fille », en singeant l'ignorance, l'innocence, voire en essayant de susciter l'apitoiement devant les policiers, leur a plus d'une fois permis de se tirer d'une situation problématique. Ainsi Marina raconte comment sa sœur Alice s'était mise à pleurer devant les policiers après qu'elle se soit faite arrêtée au volant en état d'alcoolémie. Blondie a adopté une stratégie similaire en riant aux blagues d'un policier un peu dragueur qui l'avait arrêtée en voiture alors qu'elle « venait d'éteindre le joint ».

Il est frappant de remarquer que pour bon nombre de mes interrogées, accentuer ses stéréotypes féminins consiste souvent à se faire plus bête qu'on ne l'est. Josiane nous raconte ainsi avoir fait la «fille niaise » en se faisant interpeler par la police alors qu'elle conduisait et qu'elle avait fumé du cannabis : «j'ai fait la bébéte, la petite fille toute mignonne, et ça passe. »

Une majorité des femmes interrogées ont connu dans leur entourage des hommes incarcérés pour deal ou détention de stupéfiants, mais aucune n'a connu de femme incarcérée pour un même motif. Les femmes bénéficient aussi d'avantages plus pragmatiques : les policiers de sexe masculin ne sont pas autorisés à fouiller une femme au corps, et les policières sont plus rares que les policiers. Nos interrogées cachent donc leur produit dans leurs sous-vêtements quand elles sortent avec dans l'espace public, car elles savent que les policiers ne peuvent pas les toucher. La seule interrogée à ne pas cacher ses stupéfiants dans ses sous-vêtements est Sylvie, qui a développé une stratégie originale reposant sur une idée similaire : elle cache sa drogue dans une boîte de protections hygiéniques qu'elle met dans son sac. Les policiers ou les videurs amenés à fouiller son sac sont «gênés » et «mal à l'aise » et n'osent pas ouvrir la boîte de tampons hygiéniques.

Le préjugé que peuvent avoir les forces de l'ordre et les dealers, selon lequel le deal est une affaire d'hommes, constitue une ressource pour les actrices du trafic. Néanmoins, dans de nombreux cas, les stéréotypes de genre pesant sur les femmes dans le deal sont davantage générateurs de risques que d'outils de protection et de distinction. 
Dans les discours des interrogé.e.s, il apparaît très vite que le milieu du deal est perçu comme un «monde d'hommes » (Philippe) «vachement machiste » (Nathan, 25 ans, consommateur de cannabis et vendeur de divers produits) et «dangereux (Néo, 23 ans, consommatrice de cannabis et de psychotropes et vendeuse de cannabis). Les femmes, même dans le trafic relativement pacifié que nous étudions, doivent faire face à des risques de violences et à des stéréotypes négatifs. Elles doivent, davantage que les hommes, développer des stratégies pour ne pas être escroquées et pour s'intégrer dans le trafic.

\section{Une menace de violence constante}

Nous l'avons dit, notre terrain n'est pas très violent, comparé à d'autres types de trafics. Les dealers et dealeuses interrogées n'ont jamais été impliqués dans des activités criminelles d'un autre type. Une minorité des interrogés (deux hommes et deux femmes) déclarent avoir été confrontés personnellement à la violence physique dans un contexte de deal. Néanmoins, la violence reste un outil d'intimidation et donc une menace implicite constante. Tous les interrogés s'accordent pour dire que le milieu du deal est violent, parce qu'ils ont entendu des récits d'altercations par des connaissances, parce qu'ils se sont déjà fait menacer eux-mêmes, ou parce qu'ils ont tout simplement senti la tension inhérente à ce milieu.

Les femmes doivent donc s'intégrer dans un milieu violent en grande majorité constitué d'hommes, dont certains présupposent leur faiblesse physique. Dans certains contextes de deal avec des vendeurs ou des clients inconnus, particulièrement quand ceux-ci sont en groupes et dans des espaces publics jugés dangereux, les femmes se sentent donc davantage en insécurité que les hommes. Blondie a été amenée plusieurs fois à aller acheter de la drogue dans l'espace public, et confirme ce sentiment de danger : " un demi-regard, un demi croisement de regard, et ça part en "nan nan mais baisse les yeux, me regarde pas", et ça part... [...] C'est pour ça que je me sens pas forcément en sécurité, parce que oui, même si tu fais rien, y a quand même un risque pour qu'on vienne [...] te chercher la merde ».

Le risque permanent mais implicite de violence devient un outil d'intimidation pour qui s'estime bien armé pour l'affronter. Parfois, la menace est totalement explicite, mais elle se concrétise rarement. Marina a ainsi été menacée par un dealer à qui sa sœur Alice devait de l'argent :

«Ma sœur doit des sous à un type, le type m'a appelé moi pour foutre la pression... ça fait pas peur mais c'est quand même des petites menaces tu vois. J'sais pas, moi j'ai quand même un certain recul, je me dis que c'est plus des menaces qu'autre chose, je les prends pas forcément au sérieux. Si tu fais un calcul coût-bénéfice, ça lui couterait bien plus cher de casser la gueule à ma sœur que d'oublier ses trente euros. Il aurait bien plus de chances de se faire arrêter par les condés. »

Toutes les femmes interrogées déclarent éviter d'aller seule acheter de la drogue dans les circonstances précédemment décrites. Contrairement à d'autres contextes plus violents (comme 
celui étudié au Brésil par D. Duprez, 2012), seulement certaines y vont avec un moyen de défense (couteau, bombe lacrymogène...).

La présupposée faiblesse physique des femmes peut aussi jouer en défaveur des dealeuses : Alice et Sylvie se sont ainsi fait menacer à plusieurs reprises par des dealers rivaux ou par des clients. Philippe emploie de lui-même le terme de misogynie : "Une femme qui deale c'est pas facile, ouais ; parce que y aura des mecs misogynes ou machos qui vont avoir tendance à aller dessus, et qui vont se dire 'c'est une meuf, donc elle a pas à faire ça, j'vais la carotte”’».

Le risque d'agression sexuelle est également présent dans l'esprit des femmes interrogées, qui l'évoquent en majorité en parlant des moments de consommation. Le trafic que nous étudions n'a bien sûr rien à voir avec celui de $\mathrm{P}$. Bourgois dans le quartier d'El Barrio (Bourgois, 2001), où les viols collectifs sont monnaie courante. Les agressions sexuelles et viols dans le trafic que nous étudions sont rares, mais le risque est pris en compte par toutes les femmes rencontrées. Ainsi, il est évident pour les toutes les femmes interrogées qu'il est plus dangereux pour une femme de perdre le contrôle d'elle-même dans un moment de « défonce » en soirée, le risque implicite étant l'agression sexuelle. Rose décrit bien la prédation qui peut exister en contexte festif autour des femmes sous l'emprise de stupéfiants : "Pour avoir vu plein de fois des potes se retrouver avec des espèces de mecs un peu chelous, [...] tu sens le mec qui a juste envie de se taper la meuf parce qu'à ce moment-là elle est plus vraiment maître de ses moyens. »

Les risques auxquels font face les hommes perdant le contrôle d'eux-mêmes suite à une prise de stupéfiants seraient d'un autre ordre, selon les interrogé.e.s : tandis qu'une femme risque l'agression sexuelle, un homme risque davantage de se faire agresser physiquement ou voler. Comme le dit Cécilia, «moi j'ai vu des filles complètement défoncées à la MDMA, en boîte, où elles se faisaient tripoter dans tous les sens par les mecs et elles étaient pas au courant quoi. Elles étaient complètement inertes, [...] ils leur touchaient les seins, les fesses, [...] c'est plus facile. Un garçon, bon, euh... Tu vas lui faire le sac, les poches, mais y a pas des filles qui vont le tripoter. » Cécilia a d'ailleurs une amie qui, «trop défoncée » en rave-party, «s'est fait violer ». Une de nos interrogées a également été violée, cette fois dans un contexte qui était supposé être celui d'une vente de stupéfiants : âgée de 16 ans au moment des faits, elle est montée dans la voiture d'un garçon qui lui avait proposé de lui vendre de l'herbe. Celui-ci l'a emmené chez lui pour abuser d'elle. Il existe dans le milieu du deal de stupéfiants des préjugés quant à la lubricité des femmes sous l'emprise de stupéfiants, conduisant à une pression sexuelle de la part d'hommes autour d'elles dans les moments de consommation ${ }^{6}$. Les femmes sont toutes conscientes du risque constant d'agression sexuelle, et adaptent leur comportement individuel en fonction de celui-ci. Nous rejoignons donc ici l'étude de M. Lieber (2008) sur les rapports de genre dans l'espace public, qui décrit le viol comme un risque accepté par toutes les femmes, et donc comme un outil efficace de contrôle social.

\footnotetext{
${ }^{6}$ : Selon F. Hutton (2004), certains hommes dans le milieu du deal et de la drogue pensent que la prise de certaines substances, telles que l'ectasy ou la MDMA, augmente de manière intense la libido des consommatrices. Une minorité d'hommes présupposent donc la disponibilité et de l'envie sexuelle de la consommatrice à leur égard, et parfois également de leur amoralité et de la débauche.
} 
Au-delà des menaces et dangers physico-sexuels auxquels sont confrontées les femmes dans le deal de stupéfiants étudié, elles doivent aussi faire face à des stéréotypes négatifs (M.D.

Ludwick, S. Murphy et P. Sales, 2015). Certains hommes insérés dans le trafic considèrent que les femmes ont moins de connaissances sur les produits et les codes de ce marché illicite, qu'elles sont moins capables de négocier, de s'affirmer dans les échanges et de sortir de leur position présumée de faiblesse physique. K. A. Humbolt résume cette vision de la femme par l'idée selon laquelle elles n'auraient pas la business personnality (Humbolt, 2013). Face à la masculinité hégémonique qui fait figure de référence dans le trafic, les femmes doivent déployer des stratégies d'intégration et de valorisation de leur position. Le marché de la drogue s'avère être un marché du travail hostile aux femmes, comme tant d'autres. C'est ce que résume Marina : "[on va se dire que] la femme est moins connaisseuse, que sa drogue est moins bonne... qu'elle tient moins bien... [...] qu'on pourrait p't'être l'enculer facilement parce que c'est une fille, qu'elle est faible [...] On se dit qu'une femme qui est là-dedans, $t^{\prime}$ sais c'est comme une femme maçon ou quoi, [...] c'est pas capable de gérer, on va attendre plus d'elle. »

Sylvie raconte ainsi les questions parfois condescendantes que certains hommes lui ont posé lorsqu'elle vendait de l'herbe en grande quantité, " comme si t'as 14 ans et que [...] t'es une petite fille, quoi ». Les femmes dans le deal sont donc perçues par beaucoup d'acteurs masculins du trafic comme d'éternelles «pigeonne [s] » (Néo), des novices ad vitam eternam, peu importe leur âge ou leur position hiérarchique dans le marché. Ces préjugés se répercutent sur les femmes dealeuses, à qui selon Nathan « on fait moins confiance ». La fille dealeuse ou cliente seule, sans tutelle est donc considérée comme une irresponsable à qui on ne peut pas faire confiance (si c'est une dealeuse) ou qu'on peut facilement escroquer (si c'est une cliente).

En effet, les femmes courent plus que les hommes le risque de se faire escroquer lors d'une transaction avec un vendeur inconnu ${ }^{7}$. L'arnaque est un rite de passage dans le deal de stupéfiants de classe moyenne étudié, « tout le monde passe par là » comme dit Juliane (23 ans, consommatrice de cannabis et de psychotropes). Les dix-huit interrogés se sont tous fait escroquer au moins une fois, le plus souvent au début de leur carrière de consommateur, lorsqu'ils n'étaient pas encore au fait des normes tarifaires et qualitatives et des codes du milieu. Les façons d'escroquer dans le deal sont multiples. Mais tandis que pour un homme, le risque d'escroquerie diminue au fur et à mesure qu'il acquiert les codes et connaissances du milieu des stupéfiants, pour une femme il semble qu'il demeure plus ou moins égal.

En cas d'escroquerie, les possibilités de réactions ne sont pas innombrables. Comme l'explique Sylvie : "il te présente de la merde, tu vas dire au mec que c'est dégueulasse, il va dire "non mais t'inquiète", donc [...] si t'insistes pour dire que c'est de la merde il va commencer à s'énerver, au pire il te menacera, et [...] te dira d'aller te faire enculer, il gardera la tune et il se barrera. Comme ça arrive à n'importe quel mec, mais encore pire car il saura très bien que physiquement tu fais pas l'affaire. » Plusieurs de nos interrogées ont déjà été dans des situations porteuses de danger physique du fait d'une tentative d'escroquerie.

\footnotetext{
${ }^{7}$ : K. A. Humboldt émet une analyse semblable dans son article «Women in the marijuana industry », State University Department of Sociology, Humbolt Journal of Social Relations, n`35, 2013
} 
Les femmes ne sont néanmoins pas dépourvues de ressources face au risque plus élevé d'escroquerie auquel elles font face. Une stratégie d'évitement consiste tout simplement à ne pas acheter sa drogue à un dealer inconnu, et donc à se constituer un réseau de vendeurs de confiance. Mais pour beaucoup de consommatrices ayant développé une addiction, l'indisponibilité de leur réseau de vente amical engendre automatiquement un report sur un marché bien moins convivial : celui des dealers inconnus en appartement, voire des dealers de rue. Dans ces moments, les femmes doivent développer d'autres tactiques pour sortir de l'image de faiblesse et de méconnaissance qu'elles renvoient.

Cela passe tout d'abord par l'habillement : ainsi Juliane explique qu'elle «s'habillai[t] en $m e c »$, en empruntant des vêtements amples à son frère ou à ses amis dépourvus de tout aspect « sexy », pour se rendre «moins accessible ». Cet habillement va de pair avec une posture corporelle qu'on peut qualifier de viriliste : Rose raconte que lorsqu'elle achète à un dealer inconnu, elle « accentue une part de virilité [...], mais sans en faire trop des caisses [...], faut avoir l'air crédible ». Les femmes vont aussi pouvoir faire démonstration de leurs connaissances des codes du milieu du deal, en étalant leur culture psychotropique. Cette démonstration passe par un dialogue d'initié sur les prix et la qualité, et sur un maniement du produit (le sentir, le goûter, le toucher, le peser...) nécessitant d'avoir acquis des connaissances techniques sur les tarifs et standards de qualité en vigueur, ainsi qu'une capacité de négociation.

La négociation est indispensable si on veut éviter de se faire escroquer. Comme le dit Marina : "y a toujours l'idée que la femme, elle s'y connait moins, elle osera moins négocier, on va essayer de l'impressionner... [...] il faut que tu pallies au fait que tu sois une femme». Les femmes dans le deal doivent "plus en imposer, plus s'affirmer» (Cécilia). Sortir, à tout prix, du cliché de la fille fragile et féminine, quitte à correspondre à de nouveaux stéréotypes.

Dans l'ensemble, nos interrogés ont commencé leur consommation à un âge précoce. Tous, sauf Blondie et Philippe (qui a expérimenté le cannabis à 11 ans), ont fumé leur premier joint quand ils avaient entre 13 et 16 ans, la consommation de drogues dites dures arrivant dans les deux années suivantes (excepté Bob, qui consomme uniquement du cannabis). Les femmes que nous avons rencontrées ont donc été immergées très jeune dans le milieu du deal, et en ont retiré des apprentissages et des manières de penser. Elles ressentent un double sentiment de décalage, comme femme au sein du milieu des stupéfiants et comme consommatrice dans les moments ordinaires de la vie sociale.

Être une femme usagère : un sentiment de décalage permanent

Toutes les interrogées affirment que leur intégration au milieu des stupéfiants, et donc à un style de vie déviant, a joué un rôle majeur dans leur transformation identitaire. Toutes disent également en avoir retiré des apprentissages, ainsi qu'un sentiment de décalage quasi-permanent, à la fois vis-à-vis du milieu du deal masculin, et des autres sphères de la vie sociale (telles que la sphère professionnelle, la sphère familiale...).

\section{Le développement de compétences et de personnalités liées au milieu des stupéfiants}


De nombreux auteurs ont affirmé qu'il était nécessaire d'acquérir des compétences pour être dealer.euse (Denton et O’Malley, 2001, Rachid, 2004). En effet, être inséré.e dans le trafic de stupéfiants implique comme nous l'avons dit d'intégrer des connaissances techniques (vocabulaire psychotropique, tarifs en vigueur, éléments de reconnaissances de la qualité du produit...) mais aussi des compétences sociales. L'analyse en termes d'apprentissage et de carrière mise à jour par $\mathrm{H}$. Becker (Becker, 1985) est ici tout à fait appropriée.

En tant que femmes dans un trafic masculin, les vendeuses et consommatrices interrogées ont dû apprendre à s'imposer dans les moments de deal. Elles doivent se mettre en avant, faire preuve de poigne, de caractère, ne pas avoir peur de négocier. De fait, beaucoup parlent d'un nécessaire gain de «confiance en soi » (Marina).

Egalement, plusieurs des femmes interrogées parlent d'une capacité à reconnaître les risques, d'une vigilance et d'un pragmatisme acquis dans le milieu des stupéfiants. Ainsi Sylvie a appris « à être encore plus méfiante, [...] à anticiper plus, [...] à reconnaître les types de clients [...] donc [...] à reconnaître les types de mecs ». Marina affirme qu'elle a "été dans des situations tellement difficiles [qu'elle] peu[t] forcément gérer » et qu'elle a " acquis une force par rapport à ça».

Enfin, les femmes interrogées parlent également de connaissances sur les limites de leurs corps, du fait de la nécessité de se contrôler dans les moments de consommation. Josianne affirme ainsi qu'elle a su "écouter [son] corps » : "je prenais jamais plus que c'que j'pensais que ça pourrait me mettre en danger ».

Nous voyons donc la récurrence d'un vocabulaire qu'on peut qualifier d'empowerment ${ }^{8}$, avec les termes de «force », « confiance », «maturité », «s'affirmer », «s'imposer », « sûre de $m o i »$ qui reviennent fréquemment.

Nous pouvons donc maintenant nous demander si l'intégration à l'univers des stupéfiants a contribué à façonner des traits de personnalités «psychotropiques » chez ces femmes. Le milieu des stupéfiants constitue une sous-culture. Néanmoins, il n'est pas la seule instance de socialisation secondaire de nos femmes interrogées, qui se sont aussi socialisées à travers leurs emplois, leurs études, leurs groupes de pairs non-consommateurs... Il faut donc relativiser la prégnance du milieu des stupéfiants sur la personnalité de nos interrogées, tout en admettant que l'intégration au milieu des stupéfiants a influencé leur manière d'être et de voir le monde.

La plupart des vendeuses ou consommatrices interrogées affirme ainsi que l'aspect illicite du milieu des stupéfiants les a incité à être moins légitimiste. Blondie, qui avant d'expérimenter les produits avait une très mauvaise image du milieu des stupéfiants, explique qu'elle en a retiré "une certaine ouverture d'esprit sur un monde qui pour moi était [...] choquant $\gg$. Cécilia évoque ses rencontres avec «des gens [...] qui ont appris à vivre autrement, [...] qui vivent en camion », et sa plus grande ouverture d'esprit face à des modes de vie alternatifs.

Par ailleurs, d'autres femmes interrogées mettent surtout en avant une vision du monde plus sombre et plus négative, comme Sylvie, Sophie et Marina qui éprouvent une plus grande méfiance liée à une prise de conscience sur la violence et la malsanité humaine. Marina explique qu'elle a été spectatrice de «la décadence, [...] de choses choquantes ». Le témoignage de Sophie condense bien notre propos : "Aujourd'hui encore, même si je sors moins et que je prends moins de drogue, je sens que mon parcours dans la drogue et le deal me définit. [...] ça joue sur mon rapport aux

${ }^{8}$ : Notion également utilisée par K. A. Humbolt (2013) et F. C. Hutton (2004) 
autres, sur ma vision de la vie et de l'humain. Des fois je sais que je suis agressive trop vite. » L'intégration à l'univers des stupéfiants marque la personnalité des femmes sur le long terme.

\section{Un sentiment de décalage presque omniprésent}

Nous l'avons dit et explicité, les femmes dans le deal doivent adopter des codes masculins si elles veulent être prises au sérieux en tant que clientes et acheteuses. Elles doivent donc parfois se travestir, jouer un rôle avec lequel elles ne se sentent pas en adéquation. Elles se sentent donc un peu à part à part dans ce monde social, en tant que minorité sexuée mais aussi parfois de par leur intégration sociale.

Ainsi Juliane raconte qu'elle a rompu le contact avec certaines de ses anciennes fréquentations du monde des stupéfiants, qui la qualifiaient d' « intello » parce qu'elle poursuivait ses études. Sophie a elle aussi coupé les ponts avec des amis à elle, qui «ne comprenaient pas que [...] je pouvais plus faire la fête jusqu'à midi ». Les valeurs de notre société, que sont le travail, la performance, la stabilité, entrent en contradiction avec les valeurs du milieu des stupéfiants que sont l'amusement, la détente, l'imprévisibilité, la liberté. Il peut donc être difficile de jouer sur les deux tableaux, en étant à la fois insérée dans le monde social légitime et dans le milieu des stupéfiants. Cette idée d'une « vie dédoublée » est également abordé par A. Fontaine dans sa recherche sur les rapports entre usages de drogues et vie professionnelle (2002).

Ainsi, Marina explique qu'elle a "l'impression d'être en décalage avec les gens de mon âge, [...] d'avoir plus de recul ». Elle se sent en discordance avec les autres jeunes qui découvrent les stupéfiants au moment où elle prend de la distance avec ce milieu, tout comme Rose : "quand ils se sont mis à fumer des péts moi ça faisait hyper longtemps que j'avais commencé, quand ils se sont mis à prendre des prods ça faisait hyper longtemps que j'avais arrêté ». Elles sont partagées entre l'envie d'étaler leur expérience et le désir de rester discrètes, conscientes du choc que peut provoquer le récit de leur parcours.

Mais ce sentiment de décalage est exacerbé lorsque nos consommatrices ou dealeuses de stupéfiants font face à des individus qui réprouvent catégoriquement ce genre de pratiques. Les discours moralisateurs, qui justifient l'illégalité des stupéfiants, heurtent les femmes que nous avons interrogées. Morane raconte qu'elle a « une pote, [...] elle comprend pas pourquoi on fume, [...] elle va te faire des sermons et tout, [un] jour en soirée je lui ai dit: 'Ben écoute toi tu t'es payée des Louboutin à 4000 balles, moi ça me viendrait pas à l'esprit'». Dans bien des moments de la vie sociale (à l'école, au travail, en famille...), les femmes interrogées ont le sentiment de devoir cacher cette facette de leur existence. Marina dit avoir le sentiment de "vivre une double vie », en changeant sa manière de parler, de s'habiller, de se comporter, selon si elle se trouve dans le milieu des stupéfiants ou dans une sphère sociale légitime.

Les femmes que nous avons rencontrées doivent donc très souvent dissimuler un aspect de leur vie ou de leur personnalité. Notre analyse remet ainsi en cause certains travaux des années 80 affirmant que les femmes ne peuvent pas adapter leur personnalité à la culture du deal et de la drogue (Adler, 1985), et des recherches contemporaines affirmant que l'organisation sociale du trafic de drogues n'est réellement présente que dans les banlieues.

\section{Conclusion}

La position des femmes dans le trafic bordelais étudié est ambivalente, toujours sujette à des risques de violence, de menaces, d'agressions sexuelles, d'escroqueries et à des déconsidérations. Elle est parallèlement génératrice d'avantages, les femmes dans le deal étant perçues par beaucoup 
comme des objets de séduction qu'on peut légitimement aborder en utilisant la drogue comme argument, et comme des créatures plus douces et rassurantes. Cela leur permet quand elles sont dealeuses de se démarquer face à la concurrence. Les femmes sont aussi bien plus protégées que les hommes des risques policiers, qui cible davantage leurs congénères masculins.

Ces dangers et avantages sont générateurs de diverses stratégies, allant de l'instrumentalisation de préjugés (en accentuant les traits physiques ou moraux jugés typiquement féminins) à des stratégies de protection et de défense copiant les comportements masculins (en portant des vêtements « de garçon », en adoptant une parole et une attitude corporelle viriliste, voire en s'armant). L'entrée dans le monde des drogues est une période aux forts effets socialisateurs, génératrice d'apprentissages et de compétences spécifiques. Les femmes interrogées ont ainsi le sentiment de vivre dans un double décalage, qui peut introduire une impression de différence avec les autres jeunes de leur entourage.

Ainsi, nous concluons en affirmant, comme le font M. D. Ludwick, S. Murphy et P. Sales (2015), que le genre dans le deal est à la fois un facteur de protection et de vulnérabilité. Des stéréotypes et des risques particuliers agissent sur les femmes, tout comme dans le reste de la société. Mais au sein d'un marché et d'une activité illicite comme l'est le trafic des stupéfiants, aucune force supérieure régulatrice n'intervient pour égaliser les conditions entre hommes et femmes. En revanche, notre étude montre qu'il convient de relativiser l'idée selon laquelle le trafic de stupéfiants serait seulement une instance de désocialisation, puisqu'il apparaît que les femmes en retirent des compétences qu'elles utilisent à d'autres moments de la vie sociale. Ainsi plusieurs interrogées affirment que l'assurance, l'aisance oratoire et les capacités de négociation qu'elles ont acquises leur ont, par exemple, permis d'aller plus facilement vers des groupes de gens inconnus ou de négocier un contrat de travail. Il serait intéressant d'étudier plus en profondeur la transférabilité potentielle de ces compétences, acquises dans un monde illicite et réutilisées dans les sphères légitimes de la vie sociale. On peut aussi projeter cette réflexion dans d'autres contextes particuliers impliquant des contraintes différentes : on pense par exemple aux femmes incarcérées, ou aux femmes résidant dans des grands ensembles urbains.

\section{Bibliographie :}

Adler P A : « Wheeling and Dealing - an ethnography of an upper-level drug dealing and smuggling community » - in Columbia University Press (1985)

Barbier K : «Accessoires. L'invisibilisation des femmes dans les procédures pénales en matière de stupéfiants » - in Sociologie, Université Paris-Saclay (2016)

Beck François, Peretti-Watel Patrick, Legleye Stéphane : Les usages sociaux des drogues - PUF (2007)

Beck F, Obradovic I : « Jeunes femmes sous influence. Une féminisation du public reçu pour usage de cannabis dans les dispositifs d'aide ? » - in Travail, genre et société 29 (1), $105: 127$ (2013)

Becker Howard : Outsiders. Etudes de sociologie de la déviance - Métaillé, 1985

Ben Lakdhar C : «L'économie du deal. Comprendre pour agir » - in Les Cahiers Dynamiques 56 (3), 50-57 (2012) 
Ben Lakdhar C, Weinberger D : « Du marché du cannabis au marché du THC en France implications pour le système d'offre et les politiques de lutte contre les trafics illicites de stupéfiants » - in Revue Française de Socio-Economie 1 (7), 123 :145 (2011)

Bourgois P : En quête de respect. Le crack dans les ghettos américains - Seuil, Liber (2001)

Butler $\mathrm{J}:$ «Performative Acts and Gender Constitution : an essay in phenomenology and feminist theory » - in Performing Feminisms : Feminist Critcal Theory and Theatre (1990)

Chantelat $\mathrm{P}$, Bayle E, Ferrand $\mathrm{C}:$ «Les représentations de l'activité des femmes dirigeantes dans les fédérations sportives françaises : effets de contexte et ambivalences » - in Staps 66 (4), 143 :159 (2004)

Coppel A : «Policiers contre dealers. Spirales de la violence » - in Vacarme 64 (3), 50-62 (2013)

Denton B, O’Malley P : « Gender, trust and business : women drug dealers in the illicit economy » in Brit. J. Criminol 39 (4) (1999)

Denton B, O’Malley P : « Property Crime and Women Drug Dealers in Australia » - in Journal of Drug Issues 31 (2), $465: 486$ (2001)

Fontaine A : «Usages de drogues et vie professionnelle. Recherche exploratoire. » - in Observatoire Français des Drogues et Toxicomanies (2002)

Hutton F : «Risky business : Gender, drug dealing and risk » - in Addiction Research and Theroy 13 (6), $545: 554$ (2005)

Hutton F : «Up for it, mad for it? Women, drug use and participation in club scenes » - in Health, Risk and Society 6 (3) (2004)

Humbolt K A : «Women in the marijuana industry » - in Journal of Social Relations (35) (2013)

Lieber M : «Genre, violences et espaces publics. La vulnérabilité des femmes en question » - in Presse de Sciences Po (P.F.N.S.P) (2008)

Lindstad S : « Women dealers empowered by sealing drugs [compte-rendu de la thèse de Heidi Grundetjern » - in Kilden Gender Research (2017)

Louey S, Schültz G : «Les effets de la mixité au prisme du corps et de la sexualité. Les hommes dans les métiers d'accueil » - in Travail et Emploi 140, $5: 19$ (2014)

Ludwick M D, Murphy S, Sales P : « Savvy sellers : dealing drugs, doing gender and doing difference $»$ - in Subst Use Misuse, 708 :720 (2015)

Miller J : «Gender and power on the street. Street prostitution in the era of crack cocaine » - in Sage Journal 23 (4), 427 :452 (1995)

Niget D : « chap. 16 'Bad girls'. La violence des filles : généalogie d'une panique morale » - in Penser la violence des femmes, La Découverte, 300 : 310 (2012) 
Nouguez E : «Réseaux, capital social et profit dans le deal de cannabis (enquête) » - in Terrain et Travaux 4 (1), $56: 81$ (2003)

Obradovic I : « Trente ans de réponse pénale à l'usage de stupéfiant 》 - in Observatoire Français des Drogues et Toxicomanie (2015)

Rachid (pseudo) : «Génération Scarface - la place du trafic dans une cité de la banlieue parisienne » - in Déviance et Société 28 (1), 115 : 132 (2004)

Sznitman R. S : «Drugs and gender »- in Nordic Studies on Alcohol and Drug 24, 107 : 126 (2007) Thomas O : «Pourquoi la femme aujourd'hui ? » - in Psychotropes 19 (3), 61 :74 (2013)

Trébert B : «A 74 ans, elle convoyait de la drogue par amour » - in Le Parisien - rubrique « Faits divers » (13 décembre 2016)

West C, Zimmerman D H : «Accounting for doing gender »- in Gender and Society (2009)

\section{Annexes :}

$\underline{\text { Tableau } 1 \text { - tableau récapitulatif des femmes interrogées }}$

\begin{tabular}{|c|c|c|c|c|c|c|c|c|c|c|c|}
\hline & Sophie & Marina & Alice & Julianne & Rose & Néo & Josianne & Blondie & Morane & Cécilia & Sylvie \\
\hline Âge & 21 & 23 & 26 & 23 & 29 & 23 & 26 & 27 & 24 & 24 & 22 \\
\hline $\begin{array}{l}\text { Quartier de } \\
\text { résidence }\end{array}$ & Bergonié & Capucins & Capucins & Gambetta & $\begin{array}{l}\text { Rue de } \\
\text { Bègles }\end{array}$ & $\begin{array}{l}\text { Rue de } \\
\text { Bègles }\end{array}$ & Talence & Pessac & Talence & Nansouty & $\begin{array}{l}\text { Jardin } \\
\text { Public }\end{array}$ \\
\hline $\begin{array}{l}\text { Profession } \\
\text { et revenus } \\
\text { du père }\end{array}$ & $\begin{array}{l}\text { Cadre à } \\
\text { La Poste } \\
-2000 €\end{array}$ & $\begin{array}{l}\text { Chef } \\
\text { d'entrepris } \\
\mathrm{e}-15 \\
000 €\end{array}$ & \begin{tabular}{|l|} 
Chef \\
d'entrepris \\
$e-15$ \\
$000 €$
\end{tabular} & $\begin{array}{l}\text { PDG - } \\
5000 €\end{array}$ & $\begin{array}{l}\text { Médecin - } \\
5000 €\end{array}$ & $\begin{array}{l}\text { A la } \\
\text { retraite, } \\
\text { ancien } \\
\text { inspecteu } \\
\text { r des } \\
\text { finances } \\
-3000 €\end{array}$ & $\begin{array}{l}\text { Ingénieur - } \\
3000 €\end{array}$ & $\begin{array}{l}\text { A la } \\
\text { retraite, } \\
\text { ancien } \\
\text { fonctionn } \\
\text { aire chez } \\
\text { Orange - } \\
1500 €\end{array}$ & $\begin{array}{l}\text { Menuisier } \\
-1300 €\end{array}$ & $\begin{array}{l}\text { Dans le } \\
\text { bâtiment } \\
\text { - revenus } \\
\text { inconnus }\end{array}$ & $\begin{array}{l}\text { Charpenti } \\
\text { er et } \\
\text { dealer de } \\
\text { cannabis } \\
\text { - revenus } \\
\text { indétermi } \\
\text { nés }\end{array}$ \\
\hline \multirow[t]{2}{*}{$\begin{array}{l}\text { Profession } \\
\text { et revenus } \\
\text { de la mère }\end{array}$} & $\begin{array}{l}\text { Cadre } \\
\text { commerci } \\
\text { ale pour } \\
\text { une }\end{array}$ & $\begin{array}{l}\text { Directrice } \\
\text { d'une } \\
\text { pension } \\
\text { pour }\end{array}$ & $\begin{array}{l}\text { Directrice } \\
\text { d'une } \\
\text { pension } \\
\text { pour }\end{array}$ & $\begin{array}{l}\text { Professeu } \\
\mathrm{r} \quad \text { dans } \\
\text { une } \\
\text { associatio } \\
\mathrm{n}-1300 €\end{array}$ & $\begin{array}{l}\text { Médecin - } \\
3000 €\end{array}$ & $\begin{array}{l}\text { Agente } \\
\text { des } \\
\text { impôts - } \\
1400 €\end{array}$ & $\begin{array}{l}\text { Blanchisse } \\
\text { use - } \\
1200 €\end{array}$ & $\begin{array}{l}\text { Cadre - } 2 \\
200 €\end{array}$ & Inconnue & $\begin{array}{l}\text { Assistante } \\
\text { maternell } \\
\mathrm{e}-1 \\
600 €\end{array}$ & $\begin{array}{l}\text { Auxiliaire } \\
\text { de vie et } \\
\text { dealeuse } \\
\text { de }\end{array}$ \\
\hline & $\begin{array}{l}\text { marque de } \\
\text { sport - } \\
3000 €\end{array}$ & $\begin{array}{l}\text { chevaux - } \\
200 €\end{array}$ & $\begin{array}{l}\text { chevaux - } \\
200 €\end{array}$ & & & & & & & & $\begin{array}{l}\text { cannabis - } \\
\text { revenus } \\
\text { indétermi } \\
\text { nés }\end{array}$ \\
\hline $\begin{array}{l}\text { Situation } \\
\text { profession } \\
\text { nelle }\end{array}$ & $\begin{array}{l}\text { Etudiante } \\
\text { en master }\end{array}$ & $\begin{array}{l}\text { Etudiante } \\
\text { en master }\end{array}$ & Barman & $\begin{array}{l}\text { Etudiante } \\
\text { en master }\end{array}$ & $\begin{array}{l}\text { En } \\
\text { recherche } \\
\text { d'emploi }\end{array}$ & $\begin{array}{l}\text { Etudiante } \\
\text { en master }\end{array}$ & $\begin{array}{l}\text { Vendeuse } \\
\text { en } \\
\text { boulangeri } \\
\mathrm{e}\end{array}$ & $\begin{array}{l}\text { Conductri } \\
\text { ce de bus } \\
\text { et de } \\
\text { limousine } \\
\text { s }\end{array}$ & $\begin{array}{l}\text { Animatric } \\
\text { e en centre } \\
\text { de loisirs }\end{array}$ & $\begin{array}{l}\text { Employée } \\
\text { polyvalen } \\
\text { te dans un } \\
\text { château }\end{array}$ & $\begin{array}{l}\text { Etudiante } \\
\text { en master }\end{array}$ \\
\hline $\begin{array}{l}\text { Revenus } \\
\text { mensuels } \\
\text { légaux }\end{array}$ & $600,00 €$ & $\begin{array}{l}\text { Entre } 500 \\
\text { et } 2000 €\end{array}$ & $\begin{array}{l}1500,00 \\
€\end{array}$ & $400,00 €$ & $650,00 €$ & $300,00 €$ & $1100,00 €$ & $\begin{array}{l}1400,00 \\
€\end{array}$ & $\begin{array}{l}\text { Entre } 500 \\
\text { et } 1300 €\end{array}$ & $\begin{array}{l}1100,00 \\
€\end{array}$ & $300,00 €$ \\
\hline $\begin{array}{l}\text { Place et } \\
\text { rôle au sein } \\
\text { du trafic }\end{array}$ & \begin{tabular}{|l|} 
Cliente, \\
autrefois \\
revendeus \\
e
\end{tabular} & Cliente & $\begin{array}{l}\text { Cliente, } \\
\text { autrefois } \\
\text { vendeuse }\end{array}$ & $\begin{array}{l}\text { Cliente, } \\
\text { autrefois } \\
\text { revendeus } \\
\text { e }\end{array}$ & Cliente & $\begin{array}{l}\text { Cliente, } \\
\text { autrefois } \\
\text { revendeu } \\
\text { se }\end{array}$ & $\begin{array}{l}\text { Cliente, } \\
\text { autrefois } \\
\text { revendeuse }\end{array}$ & Cliente & Cliente & $\begin{array}{l}\text { Cliente } \\
\text { autrefois } \\
\text { vendeuse }\end{array}$ & \\
\hline
\end{tabular}




\begin{tabular}{|c|c|c|c|c|c|c|c|c|c|c|}
\hline $\begin{array}{l}\text { Drogues } \\
\text { consommé } \\
\text { es }\end{array}$ & $\begin{array}{l}\text { Cannabis } \\
\text { kétamine } \\
\text { champign } \\
\text { ons } \\
\text { héroïne } \\
\text { cocaïne } \\
\text { MDMA } \\
\text { speed } \\
\text { opium } \\
\text { LSD } \\
\text { mescaline } \\
\text { drogues de } \\
\text { synthèse }\end{array}$ & $\begin{array}{l}\text { Cannabis } \\
\text { kétamine } \\
\text { cocaïne } \\
\text { MDMA } \\
\text { speed LSD } \\
\text { champign } \\
\text { ons } \\
\text { mescaline }\end{array}$ & $\begin{array}{l}\text { Cannabis } \\
\text { kétamine } \\
\text { cocaïne } \\
\text { MDMA } \\
\text { speed LSD } \\
\text { mescaline } \\
\text { champign } \\
\text { ons } \\
\text { drogues de } \\
\text { synthèses }\end{array}$ & $\begin{array}{l}\text { Cannabis } \\
\text { ectasy } \\
\text { LSD } \\
\text { kétamine } \\
\text { MDMA }\end{array}$ & $\begin{array}{l}\text { Cannabis } \\
\text { champign } \\
\text { ons } \\
\text { cocaïne } \\
\text { speed } \\
\text { MDMA }\end{array}$ & $\begin{array}{l}\text { Cannabis } \\
\text { ectasy } \\
\text { LSD } \\
\text { kétamine } \\
\text { champig } \\
\text { nons } \\
\text { speed } \\
\text { MDMA }\end{array}$ & $\begin{array}{l}\text { Cannabis } \\
\text { MDMA } \\
\text { cocaïne } \\
\text { kétamine } \\
\text { champigno } \\
\text { ns } \\
\text { LSD }\end{array}$ & $\begin{array}{l}\text { Cannabis } \\
\text { MDMA } \\
\text { ectasy } \\
\text { LSD }\end{array}$ & $\begin{array}{l}\text { Cannabis } \\
\text { MDMA } \\
\text { LSD } \\
\text { cocaïne }\end{array}$ & $\begin{array}{l}\text { Cannabis } \\
\text { LSD } \\
\text { kétamine } \\
\text { MDMA } \\
\text { ectasy } \\
\text { speed } \\
\text { cocaïne } \\
\text { drogues de } \\
\text { synthèse }\end{array}$ \\
\hline $\begin{array}{l}\text { Drogues } \\
\text { vendues }\end{array}$ & $\begin{array}{l}\text { Cannabis, } \\
\text { LSD, } \\
\text { MDMA }\end{array}$ & & $\begin{array}{l}\text { Cannabis, } \\
\text { LSD, } \\
\text { mescaline } \\
\text {, MDMA }\end{array}$ & Cannabis & & Cannabis & MDMA & & & $\begin{array}{l}\text { Kétamine, } \\
\text { cannabis, } \\
\text { MDMA }\end{array}$ \\
\hline
\end{tabular}

$\underline{\text { Tableau } 2 \text { - tableau récapitulatif des interrogés hommes }}$

\begin{tabular}{|c|c|c|c|c|c|}
\hline & Alain & Nathan & Gilles & Bob & Philippe \\
\hline Âge & 27 & 25 & 28 & 23 & 25 \\
\hline $\begin{array}{l}\text { Quartier de } \\
\text { résidence }\end{array}$ & Saint Genès & Bergonié & Saint Genès & Saint Michel & Capucins \\
\hline $\begin{array}{l}\text { Profession et } \\
\text { revenus du } \\
\text { père }\end{array}$ & $\begin{array}{l}\text { Fabricant de } \\
\text { moteur }-1 \\
600 €\end{array}$ & Inconnu & $\begin{array}{ll}\text { Patron } & \text { de } \\
\text { restaurant - } & \\
5000 € & \end{array}$ & $\begin{array}{l}\text { Ouvrier du } \\
\text { bâtiment }-1 \\
500 €\end{array}$ & $\begin{array}{l}\text { Gérant d'une } \\
\text { société } \\
\text { d'hôtellerie } \\
\text { restauration }-8 \\
000 €\end{array}$ \\
\hline $\begin{array}{l}\text { Profession et } \\
\text { revenus de la } \\
\text { mère }\end{array}$ & $\begin{array}{l}\text { En maison de } \\
\text { retraite }-1 \\
300 €\end{array}$ & $\begin{array}{lr}\text { Salariée } & \\
\text { dans } & \text { un } \\
\text { institut } & \text { de } \\
\text { sondage } & -1 \\
500 € & \\
\end{array}$ & $\begin{array}{l}\text { Saisonnière - } \\
\text { revenu } \\
\text { indéterminé }\end{array}$ & Métier inconnu & $\begin{array}{l}\text { Employée dans } \\
\text { la société de } \\
\text { restauration - } \\
2000 €\end{array}$ \\
\hline $\begin{array}{l}\text { Situation } \\
\text { professionnelle }\end{array}$ & Chômeur & Réceptionniste & Cuisinier & $\begin{array}{l}\text { Employé } \\
\text { fast food }\end{array}$ & Cuisinier \\
\hline $\begin{array}{l}\text { Revenus } \\
\text { mensuels } \\
\text { légaux }\end{array}$ & $\begin{array}{l}\text { Entre } 800 \text { et } \\
1000 €\end{array}$ & $1400,00 €$ & $1400,00 €$ & $1200,00 €$ & $1600,00 €$ \\
\hline $\begin{array}{l}\text { Place et rôle au } \\
\text { sein du trafic }\end{array}$ & Client & $\begin{array}{l}\text { Client, ancien } \\
\text { vendeur }\end{array}$ & $\begin{array}{l}\text { Client, ancien } \\
\text { revendeur }\end{array}$ & $\begin{array}{l}\text { Client, ancien } \\
\text { vendeur }\end{array}$ & $\begin{array}{l}\text { Client, ancien } \\
\text { vendeur }\end{array}$ \\
\hline
\end{tabular}




\begin{tabular}{|l|l|l|l|l|l|}
$\begin{array}{l}\text { Drogues } \\
\text { consommées }\end{array}$ & $\begin{array}{l}\text { Cannabis, } \\
\text { MDMA, } \\
\text { cocaïne, } \\
\text { kétamine, LSD, } \\
\text { ecstasy, speed }\end{array}$ & $\begin{array}{l}\text { Cannabis, LSD, } \\
\text { cocaïne, } \\
\text { ecstasy, salvia } \\
\text { divinarium, } \\
\text { kétamine, speed }\end{array}$ & $\begin{array}{l}\text { Cannabis, } \\
\text { MDMA, } \\
\text { champignons, } \\
\text { LSD, cocaïne, } \\
\text { héroïne, } \\
\text { kétamine, } \\
\text { mescaline, } \\
\text { héroïne, opium, } \\
\text { méthoxétamine } \\
\text { spee }\end{array}$ & $\begin{array}{l}\text { Cannabis } \\
\text { MDMA, LSD, } \\
\text { kétamine, } \\
\text { mescaline, } \\
\text { speed, cocaïne }\end{array}$ \\
\hline $\begin{array}{l}\text { Drogues } \\
\text { vendues }\end{array}$ & & $\begin{array}{l}\text { Cannabis, } \\
\text { drogues de } \\
\text { synthèse }\end{array}$ & $\begin{array}{l}\text { Cannabis, } \\
\text { MDMA }\end{array}$ & Cannabis & $\begin{array}{l}\text { Cannabis, } \\
\text { cocaïne }\end{array}$ \\
\hline
\end{tabular}

